\title{
Exploring the Landscape of Brain States
}

Włodzisław Duch \& Krzysztof Dobosz (Nicolaus Copernicus University, Toruń, Poland),

Aleksandar Jovanovic (University of Belgrade, Serbia),

W. Klonowski (Nałęcz Institute of Biocybernetics \& Biomedical Eng, Polish Academy of Sciences, Warsaw, Poland).

\section{Introduction}

The goal is to understand neurodynamics, identify attractor states, their properties, understand how neural processes give rise to attention and conscious mental states during spontaneous thinking or mind wandering.

Using trained, biologically plausible model of neural network that has learned associations between phonology, orthography and semantic of many words, implemented in the Emergent simulator, we are investigating the landscape of attractor states using fuzzy symbolic dynamics visualization and recurrence plots.

Hypothesis: transient states are not perceived in a conscious way, perception requires formation of a quasi-stable state where trajectory of the system falls into basin of some attractor,

transitions between attractors generate "stream of consciousness".

\section{Model of Reading}

Emergent neural simulator:

Aisa, B., Mingus, B., and O'Reilly, R. The emergent neural

modeling system. Neural Networks 21, 1045-1212, 2008.

3-layer model of reading: orthography, phonology, semantics, or distributio of activity over 140 microfeatures of concepts Hidden layers in between Learning: mapping one of the 3 layers to the other two. Fluctuations around final configuration show basins of attractors representing concepts. How to see their properties and relations?

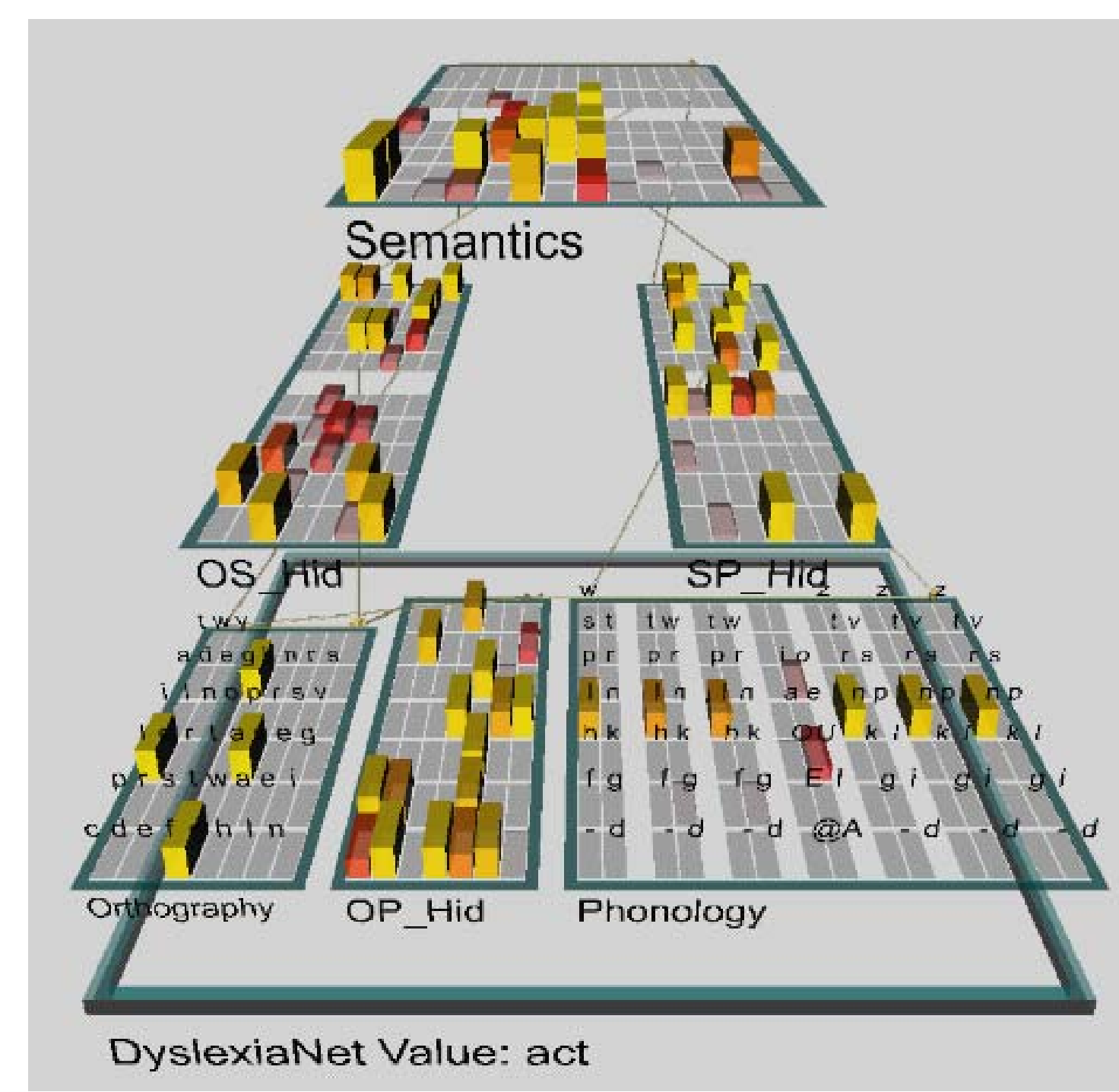

Trajectories of the activity of 140 neurons in the semantic layer subject to synaptic noise and neural fatigue, oscillate in attractor basins that correspond to word semantics. Trajectories of 4 pairs of correlated words are shown, with concrete words in the first two pairs (flag, coat hind deer) marked in blue, and abstract words (wage, coat, loss, gain) as the last two pairs, marked in red-yellow.



\section{Trajectories are good for ...}

New area in psycholinguistics: investigation of dynamical cognition, influence of masking on semantic and phonological errors, tempora dynamics in perception, effects of priming, associations, continuous mental trajectories, formation of mems and their influence on thinking and relating mental states to neural/brain properties. M. Spivey, Continuity of mind. Oxford University Press 2007. P. McLeod, T. Shallice, D.C. Plaut, Attractor dynamics in word recognition: converging evidence from errors by normal subjects, dyslexic patients and a connectionist model. Cognition 74, 91-113, 2000

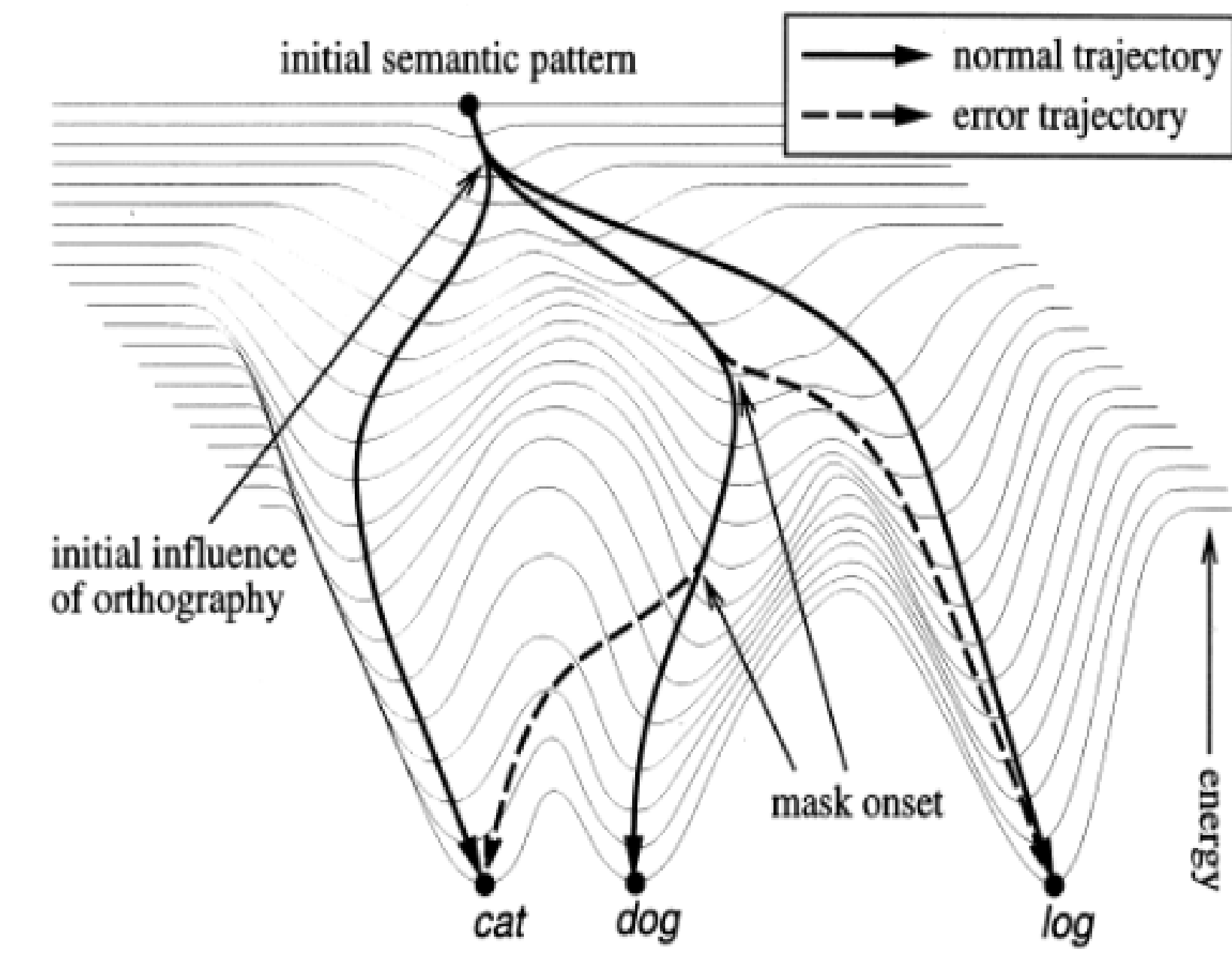

\section{Recurrence Plots}

Eckmann et al. (1987) introduced recurrence plots, a general method for analysis of dynamical systems

A trajectory $x_{i}=x\left(t_{i}\right)$ returns to almost

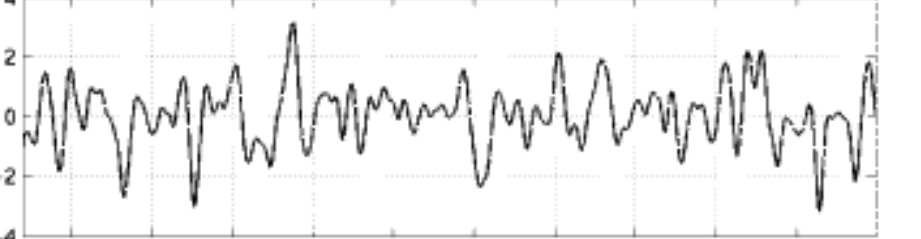
the same area (within $\varepsilon$ distance) at some other point in time, creating non-zero elements (or black dots in the plot) of the recurrence matrix:

$$
\begin{gathered}
R_{i j}(\varepsilon)=\Theta\left(\varepsilon-\mid x_{i}-x_{j}\right) \\
\Theta(z)= \begin{cases}1 & z \geq 0 \\
0 & z<0\end{cases}
\end{gathered}
$$

With some experience such plots allow for identification of many interesting behaviors of the system. However, these plots depend strongly on the choice of $\varepsilon$ and the choice of the time step $\Delta t=t_{i+1}-t_{i}$

In the analysis of high-dimensional dynamical systems it is better to plot all distances between

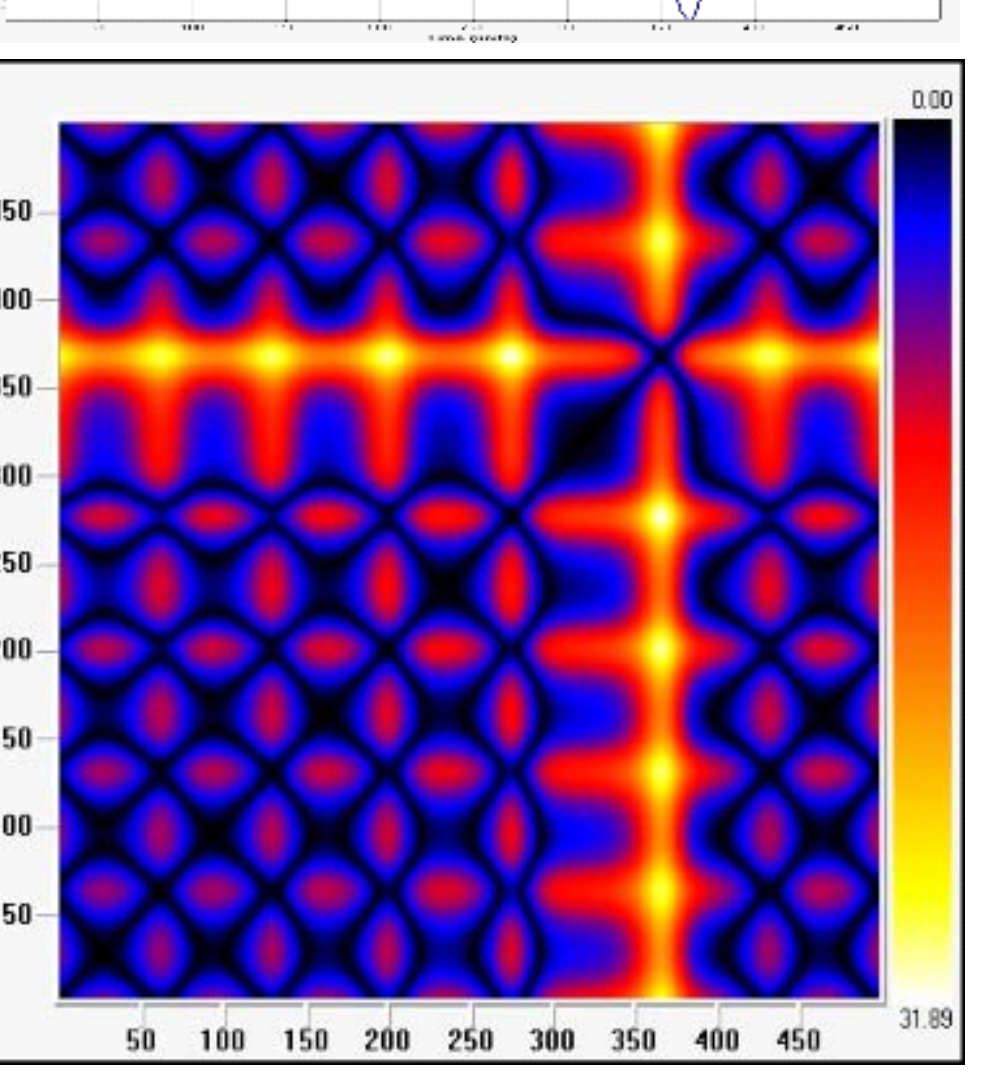
trajectory points using color scale for distance determination. The recurrence matrix is given by:

$$
R_{i j}=x_{i}-x_{j}
$$

Such recurrence plots may be smoothed using non-linear distance function $\|\cdot\|$, capturing essential info about system's behavior, especially its attractor dynamics.

Recurrence plots have wide applications for analysis of time series, including high-dimensional neurodynamical systems, but their interpretation is not always easy; FSD complements them.

\section{Fuzzy Symbolic Dynamics}

Fuzzy Symbolic Dynamics (FSD) is used to:

- Reduce dimensionality of trajectory $x(t)$, visualize it in 2 or $3 \mathrm{D}$.

- Estimate properties of attractors: number, position, strength.

- Can be combined with time-frequency or component analysis.

Algorithm:

1. Standardize data.

2. Find cluster centers (e.g. by k-means algorithm): $\mu_{1}, \mu_{2}$

3. Use non-linear mapping to reduce dimensionality:

$$
y_{k}\left(t ; \mu_{k}, \Sigma_{k}\right)=\exp \left(-\left(x(t)-\mu_{k}\right)^{\mathrm{T}} \Sigma_{k}^{-1}\left(x(t)-\mu_{k}\right)\right)
$$

Reference points $\mu_{\mathrm{i}}$ may be optimized to resolve minimal distances.
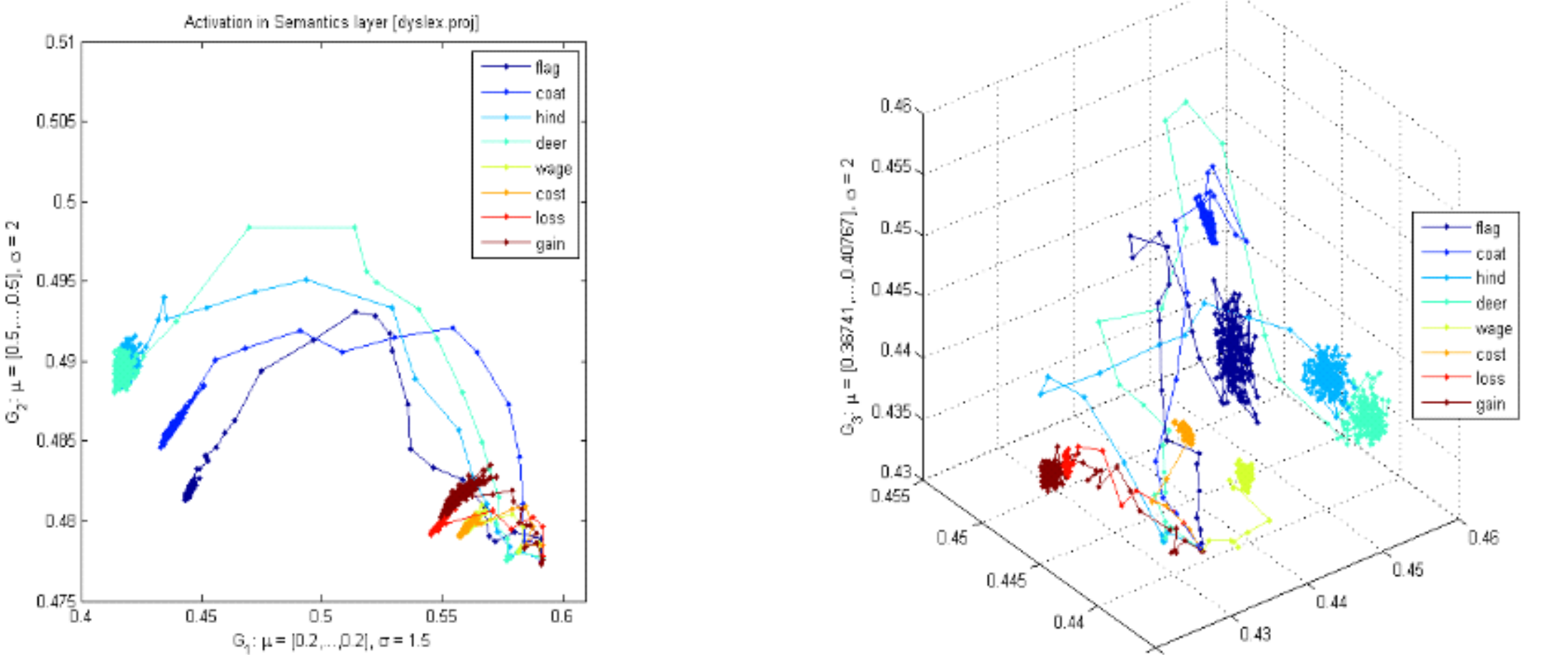

\section{Conclusions}

Spivey (2007) recommended symbolic dynamics for investigation of mental states, but recurrent plots and fuzzy symbolic dynamics may show in a much better way the continuity of mental states.

2. FSD/RP type of visualization shows the microstructure of cognition, at the conscious as well as subconscious levels, with fast transition states and

With more detailed models of neurons many phenomena related to mental states, associative thinking, attention deficits, temporo-spatial processing disorders and other mental problems may be investigated, and connected to experimental observations.

4. FSD/RP analysis may be applied to any multi-channel signals, applications to real EEG data are in progress.

\section{Recurrence Analysis}

The model of reading (used also for dyslexia) was modified adding accommodation (neural fatigue) mechanism into neuronal units based on $\mathrm{K}^{+}\left(\mathrm{Ca}^{++}\right)$ion channels, plus synaptic Gaussian noise with zero mean and 0.02 variance that provides an extra energy to facilitate free evolution.

Interesting properties can be obtained from recurrence plots:

1. Blue squares along the diagonal line show attractor basins.

2. The size of each square represents time that the system is spending in each attractor.

3. The color intensity allows for estimation of the strength of the attractor, e.g. dark blue square indicates a very strong attractor with small variance; color distribution gives some idea about the shape of attractor basin.

4. Recurrence analysis shows states to which the system is returning during evolution, displaying distance between attractors. Blue squares outside the diagonal line indicate that the distance between these two attractor basins is small, and if the square is uniformly dark blue, that the system has returned to the same basin of attraction.

5. Block structure depends mostly on accommodation parameters of neurons, attractor basins vanish when neurons are fatigues, and this facilitates jumps to distant attractors.
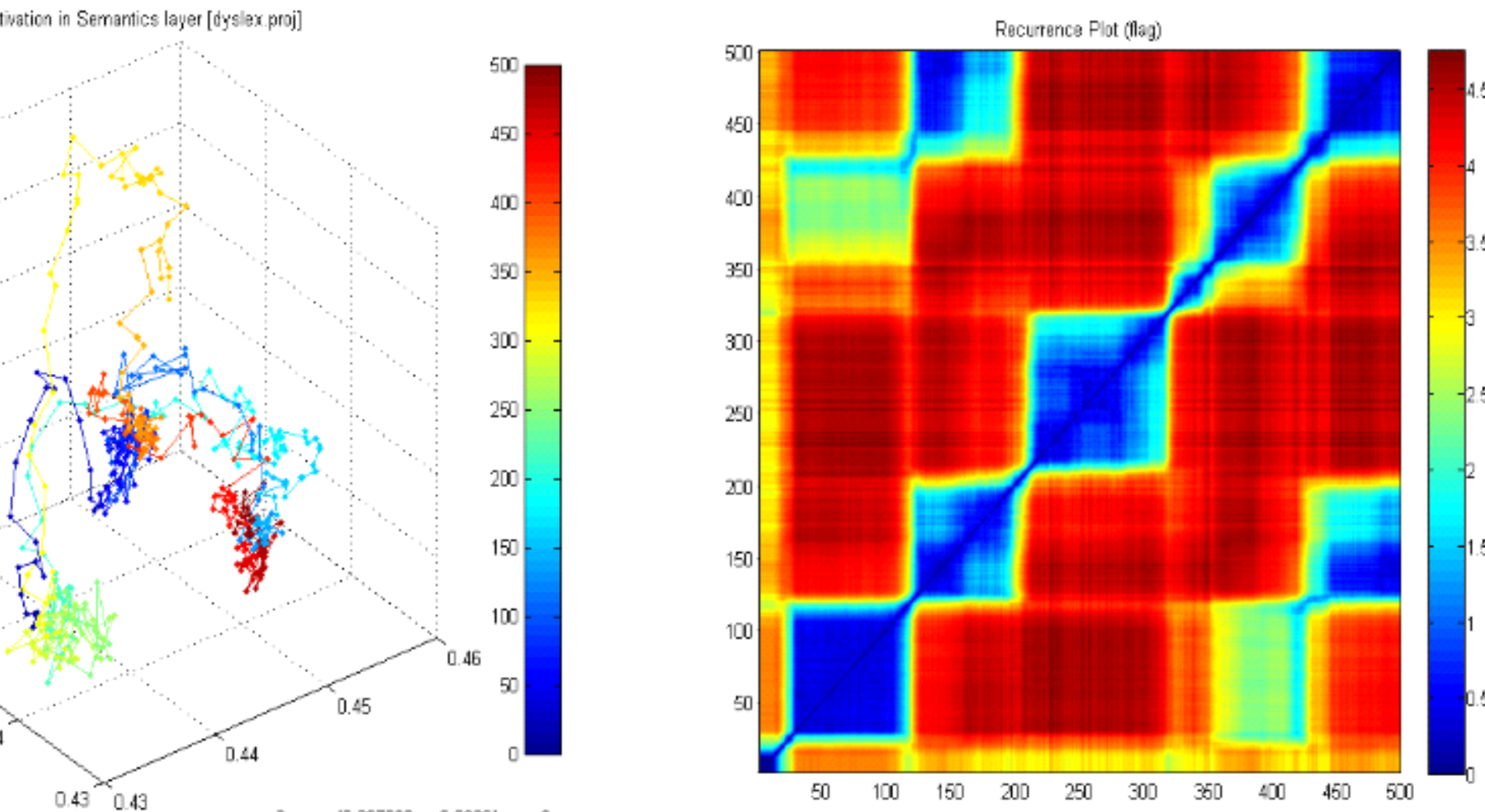

\section{Results}

Neurodynamics of this model shows many interesting properties

1. The landscape of attractor basins available for neurodynamics is not fixed, but changes depending on the history and context of the current event - the same stream is never entered twice.

2. Structure of spontaneous transitions between states of semantic network is regulated by the synaptic noise and neural accommodation, giving rise to different speed of mental time flow.

3. Depending on the network parameters (inhibition vs. excitation, neural accommodation due to leak ion channels etc.), availability of neurotransmitters, attractor basins may be deep and entrap neurodynamics, making the attention shifts difficult (leading to autism-like behavior), or it may be too shallow and the system will not be able to focus attention (ADHD-like behavior). This may be investigated by plotting the trajectory variance in attractor basin against noise variance: steep rise signifie weak attractors, while slow rise means strong (deep and narrow attractors)

4. Strong recurrent connections lead to fewer but larger basins of attraction.

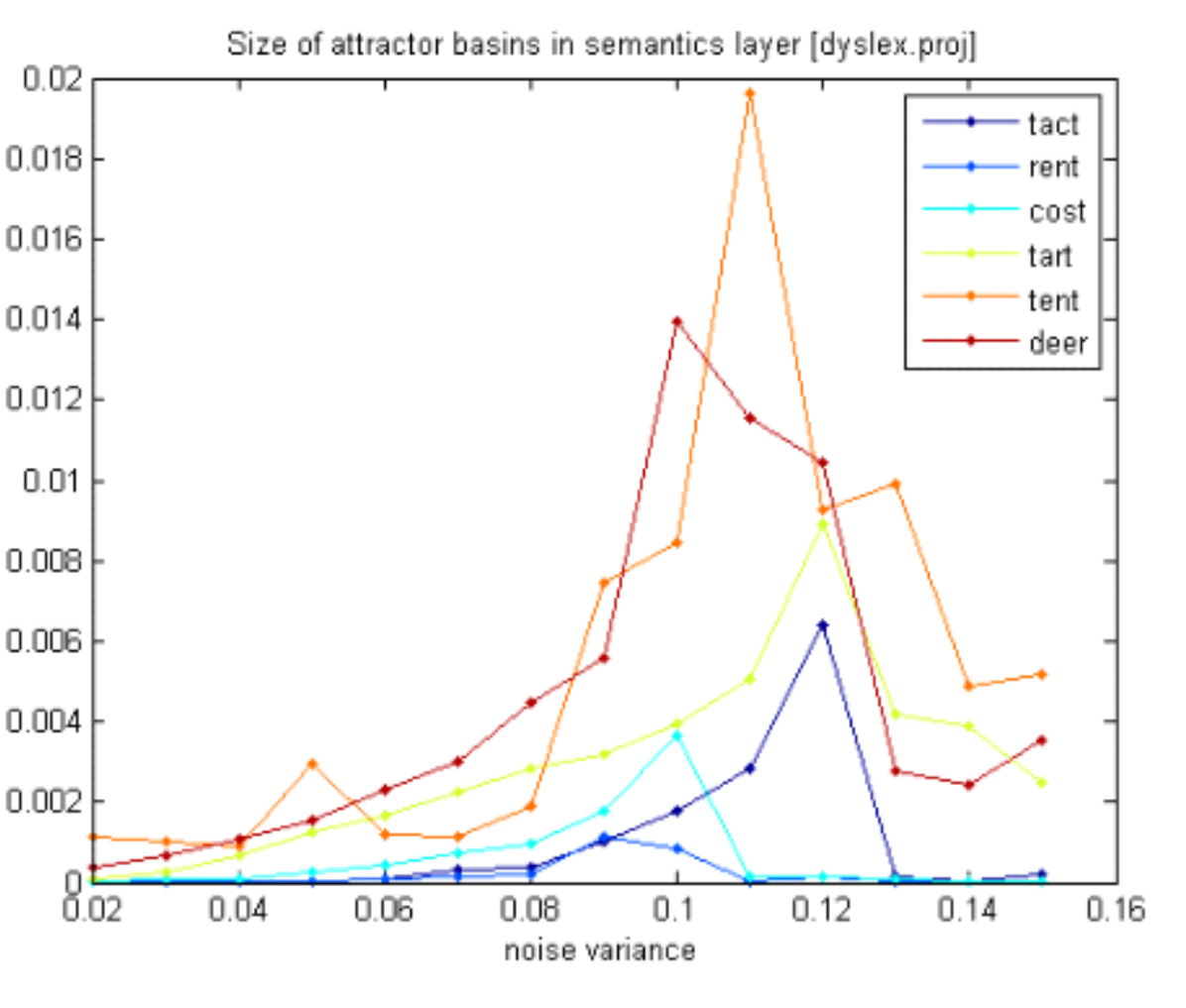

\section{References}

1. M. Spivey, Continuity of mind. Oxford University Press 2007.

2. Dobosz K, Duch W, Understanding Neurodynamical Systems via Fuzzy Symbolic Dynamics. Neural Networks (2009) http://dx.doi.org/10.1016/.neunet.2009.12.005

3. Dobosz K, Duch W, Fuzzy Symbolic Dynamics for Neurodynamical Systems. Lecture Notes in Computer Sci. 5164, 471-478, 2008

4. Dobosz K, Duch W, Global Visualization of Neural Dynamics. Neuromath Workshop, Jena, Germany, 2008, pp. 15-16

5. Duch W, Consciousness and Attention in Autism Spectrum Disorders. Coma and Consciousness. Clinical, Societal and Ethical Implications. Berlin, 4-5 June 2009, p. 46

Google: W Duch for papers/presentations 